\title{
Investigation of sorption and mechanical properties of Anacardium Occidentale $L$. exudate/polystyrene blends
}

\author{
Alisi Ikechukwu Ogadimma ${ }^{1, *}$, Gimba Casmir Emmanuel², Kolawole Emmanuel Gbadebo ${ }^{3}$ \\ ${ }^{1}$ Department of Applied Chemistry, Federal University, Dutsinma, Katsina State, Nigeria \\ ${ }^{2}$ Department of Chemistry, Ahmadu Bello University, Zaria, Kaduna State, Nigeria \\ ${ }^{3}$ Department of Industrial Chemistry, Bowen University, Iwo, Osun State, Nigeria
}

Email address:

ialisi@fudutsinma.edu.ng (A. I. Ogadimma)

\section{To cite this article:}

Alisi Ikechukwu Ogadimma, Gimba Casmir Emmanuel, Kolawole Emmanuel Gbadebo. Investigation of Sorption and Mechanical Properties of Anacardium Occidentale L. Exudate/Polystyrene Blends. American Journal of Applied Chemistry. Vol. 2, No. 5, 2014 , pp. 81-84.

doi: 10.11648/j.ajac.20140205.13

\begin{abstract}
This research employed the technique of melt blending to successfully blend commercial polystyrene (PS) with purified cashew gum (PCG) and induce biodegradation on the resulting polymer blend. Water sorption tests on the resulting polymer blends were investigated according to ASTM D0570 standard. Also tensile, hardness and charpy impact tests were conducted according to ASTM D3039, ASTM D2240-89 and ASTM 370 standards respectively. There was an observed increase in density with increase in PCG composition. The maximum density recorded was $0.996 \mathrm{gcm}^{-3}$. The water sorption analysis show that DS (degree of swelling) and WL (weight loss) increase with PCG concentration to a maximum value of $100 \%$ recorded for the sample $20 \% \mathrm{PS} / 80 \% \mathrm{PCG}$. The tensile test results indicate a general decrease in Young's modulus, tensile strength and percentage elongation with increase in the percentage of PCG. Also hardness test results based on the Rockwell F - scale indicate an improvement in mechanical strength with increase in PCG composition. The sample with composition 30\%PS/70\%PCG has the highest hardness value of $69.1 \mathrm{HRF}$. While the results of charpy impact test indicate a general decrease in impact strength as the concentration of PCG in PS increases with a minimum value of $2272.72 \mathrm{Jm}^{-2}$ for the composition $20 \% \mathrm{PS} / 80 \% \mathrm{PCG}$.
\end{abstract}

Keywords: Polystyrene, Purified Cashew Gum, Blended Samples, Sample Composition

\section{Introduction}

A polymer blend is a macroscopically homogeneous mixture of two or more different species of polymer (McNaught and Wilkinson, 1997). Polymer blends are capable of providing materials with extended useful properties beyond the range that can be obtained from single polymer equivalents (Buthaina and Karrer, 2010). Blending of polymers for property improvement or for economic advantage has gained considerable importance in the field of polymer science in the last decade (George et al., 1986).

Also, in recent time, research is focusing increasingly on the development of polymers that combine the desired functionality during use and rapid degradation after disposal as a viable alternative to conventional nondegradable polymers, mostly for applications in which long degradation times are undesirable. Biodegradable polymers fit this context perfectly, since they degrade rapidly and contain nontoxic end products which have low permanence in the environment and are completely metabolized by soil microorganisms (Scott and Gillead, 1995).

Polystyrene (PS) is a thermoplastic polymer with high heat resistance due to high melting temperature $\left(>260^{\circ} \mathrm{C}\right)$, excellent chemical resistance, high degree of crystallinity, low specific gravity, good dielectric properties, and relatively fast crystallization rate (Cimmino et al., 1991). Because of these excellent properties of PS, it can be used as a material for electronics, automotive products, and industrial films. However, the persistence of PS products in the environment after use due to its resistance to biodegradation limit its use and these drawbacks on the properties of PS can be improved by blending with cashew gum as given in this work.

A lot of research has been conducted to investigate the effect of blending PS with other polymers such as elastomers (Azman et al., 2003; Stelling et al., 1981; Zebarjad et al., 2003), high density polyethylene (HDPE) and polypropylene (PP) (Wantinee et al., (2007), Poly(methyl methacrylate) (PMMA) (Kaniappan and Latha, (2011), and Acrylonitrile- 
Butadiene-Styrene (ABS) (Buthaina and Karrer 2010). However, very little research has been centered on blending PS with naturally occuring biopolymers.

Anacardium occidentale exudate, popularly called Cashew tree gum is the biopolymer of choice in this work. Cashew gum (CG) is an exudate polysaccharide from Anacardium occidentale trees. The plant is native to Brazil and grows in many tropical and sub-tropical countries. Major cashew growing areas in Nigeria are most states in the South-East and South-West (Ezeagu, 2002).

The gum has been studied widely for various pharmaceutical applications as it is inexpensive, non-toxic, biodegradable, and possesses appropriate physicochemical characteristics (Gyedu-Akoto et al. 2008 and Kumar et al., 2009).

In this work, Cashew tree gum which was subjected to chemical purification was blended with PS of laboratory grade. These blends were prepared through melt mixing technique using the two-roll mill maintained at appropriate processing conditions. This was followed by compression molding of the blended samples using a mould engraved with standard sample dimensions. The mechanical properties were investigated by tensile, hardness and charpy impact tests according to ASTM D3039, ASTM D2240-89 and ASTM 370 standards respectively. Also the chemical resistance of the polymer blends produced were investigated by sorption tests according to ASTM D0570 standard.

\section{Experimental}

\subsection{Materials and Equipment}

Table 1. List of materials and their corresponding grades and source

\begin{tabular}{lll}
\hline Material & Grade & Source \\
\hline $\begin{array}{l}\text { Polystyrene, PS (pellet) } \\
\text { Anacardium occidentale gum } 280000\end{array}$ & Natural exudates & $\begin{array}{l}\text { Plantation garden } \\
\text { in Ahmadu Bello } \\
\text { University Zaria }\end{array}$ \\
$\begin{array}{l}\text { Ethanol, Benzene, Chloroform, } \\
\text { Carbon Tetrachloride, } \\
\begin{array}{l}\text { Cyclohexane, Cyclohexanone, } \\
\text { Petroleum Ether, } \\
\text { Tetrahydrofuran, Toluene, 1,4- } \\
\text { Dioxane }\end{array}\end{array}$ & Analytical & Aldrich \\
\hline
\end{tabular}

Table 1 gives a list of the materials used in this work together with their corresponding grades and sources.

The equipment used in this work and their corresponding models are listed in table 2 .

Table 2. List of equipment used and their corresponding models.

\begin{tabular}{ll}
\hline Equipment & Model \\
\hline General Laboratory Centrifuge & SORVIAL75066180 \\
pH meter & Jenway 3505 \\
Infrared spectrophotometer & Shimadzu FTIR-8400S \\
Two-roll mill & 5183 \\
Tensometer & Hounsfield \\
Carver Hand press & $3851-0$ \\
Charpy Impact Tester & Cat. Nr.412 \\
Indentec Universal Hardness Testing Machine & $8187.5 \mathrm{~L} \mathrm{Kv} \mathrm{model} \mathrm{B}$ \\
\hline
\end{tabular}

\subsection{Blend Formulation}

Each PS and PCG powder was thoroughly mixed together in the two-roll mill to give blends of various compositions. $30 \mathrm{~g}$ of PS/PCG blend composition was formulated. The various compositions of the blend samples were obtained as shown in table 3 below:

Table 3. PS/PCG blends composition.

\begin{tabular}{llll}
\hline Polymer & Gum (Weight \% Ratio) & Polymer & Gum (Mass Ratio) \\
\hline 100 & 0 & 30.00 & 0.00 \\
90 & 10 & 27.00 & 3.00 \\
80 & 20 & 24.00 & 6.00 \\
70 & 30 & 21.00 & 9.00 \\
60 & 40 & 18.00 & 12.00 \\
50 & 50 & 15.00 & 15.00 \\
40 & 60 & 12.00 & 18.00 \\
30 & 70 & 9.00 & 21.00 \\
20 & 80 & 6.00 & 24.00 \\
\hline
\end{tabular}

The results of 100: 0 for PS/PCG was used as control. The blending technique employed is melt blending. A laboratoryscale two-roll mill was employed for blend preparations. The samples were blended with the front roll maintained at a temperature of $175^{\circ} \mathrm{C}$ while the rear roll was at temperature of $145^{\circ} \mathrm{C}$.

The PS homopolymer was initially placed on the front roll for $3 \mathrm{~min}$ to facilitate processing. Then the roll mill was started and the PCG powder added intermittently a little at a time. The milling was continued for 5 minutes. Occasionally, the molten blend was scraped out from the roll, wrapped several times and milled back to the roll. After $5 \mathrm{~min}$ of milling, the molten blend was scraped out and cut into specimens of approximately $2 \mathrm{~cm} \times 2 \mathrm{~cm}$ in size. These pieces were then introduced into a grinding mill where small pellet-sized samples were obtained. The blended sample pellets were subjected to compression moulding on a Carver Laboratory Press equipped with temperature controllers. The mold was designed to accommodate samples with dimensions according to ASTM standard. The temperature was set at $175^{\circ}$ at a pressure of 8.5 Tons for a determined period of 8 minutes. The molded samples were then separated from the mold and labeled appropriately.

The blended samples were characterized by various analytical methods such as: absorption tests, tensile tests, impact tests and hardness tests.

\subsection{Blend Characterization}

\subsubsection{Determination of the Density of Blended Samples}

The dimensions (length, width and thickness) of the blended polymer samples were accurately measured with the aid of venier calipers while their masses were measured using an electronic weighing balance. The above measurements were carried out in order to obtain the density of each blend composition. It is also aimed at determining the effect of PCG on the density of PS. The densities of the blended samples were then compared with that of the control sample. 


\subsubsection{Absorption Test}

Water absorption tests were investigated according to ASTM D0570 standard. The polymer blends were suspended in $200 \mathrm{ml}$ of distilled water at room temperature in desiccators for a total period of 7 days with readings taken after every 24 hours. After the required amount of time the samples were removed and gently dried using a filter paper to remove water adhering to its surface. The degree of swelling (DS) was calculated using the following equation:

$$
\mathrm{DS}=\left(\frac{\mathrm{m}_{2}-\mathrm{m}_{1}}{\mathrm{~m}_{1}}\right) \times 100
$$

Similarly, the weight loss (WL) was calculated using the equation:

$$
\mathrm{WL}=\left(\frac{\mathrm{m}_{1}-\mathrm{m}_{2}}{\mathrm{~m}_{1}}\right) \times 100
$$

Where, $\mathrm{m}_{1}$ and $\mathrm{m}_{2}$ are the initial and final masses of sample respectively.

\subsubsection{Mechanical Tests}

\subsubsection{Tensile Test}

Tensile test for all the blended samples were carried out according to ASTM D3039 standard. Sample dimensions of: $100 \mathrm{~mm} \times 10 \mathrm{~mm} \times 4 \mathrm{~mm}$ length, breadth and thickness were used. The experiments were conducted on a universal loading machine type Hounsfield Tensometer of maximum capacity $20.00 \mathrm{KN}$ at room temperature. A gauge length of $30 \mathrm{~mm}$ was used. Tensile analysis of the samples was accomplished using a tensile load of $2.00 \mathrm{KN}$ at moderate strain rate until the point of failure. From the tensile analysis, force and elongation values were recorded. Young's modulus, ultimate tensile strength and percentage elongation were calculated from the resultant stress-strain curves.

\subsubsection{Hardness Test}

The mechanical strength of the samples were estimated by hardness measurements. Hardness values of the blended samples were determined according to ASTM D2240-89 standard using Indentec Hardness Testing Machine. Rockwell test (F-Scale) with 1/16 inch (steel ball) indentor was used. The minor load used was $10 \mathrm{~kg}$ while the major load used was $60 \mathrm{~kg}$ with an exposure time of 10 seconds. The hardness test on each of the blended samples was conducted at three different points distributed over the test piece to obtain mean values.

\subsubsection{Charpy Impact Test}

Charpy impact test analysis for the samples was conducted according to ASTM 370 standard using a Charpy Impact Testing Machine. In this analysis the $15 \mathrm{~J}$ capacity hammer was employed to determine the fracture energy of each sample. The impact strength of each sample was calculated using the expression:

$$
\text { Impact Strength }=\frac{\text { Fracture Energy } \text { Required }}{\text { Area of the Sample }}
$$

\section{Results and Discussions}

\subsection{Density Analysis of Sample Blends}



Fig 1. Graphical representation of PS/PCG sample blend densities against their corresponding compositions.

Sample densities of PS/PCG blends are graphically illustrated in fig.1.

Figure 1 indicates a gradual increase in the density of PS blends with increase in the composition of PCG in the sample blends. This slight increase in density is attributed to the slightly higher density value of PCG compared to PS.

\subsection{Water Sorption Test on Blended Samples}

The results of water sorption tests conducted on PS/PCG blend samples are given in figures 2. Figure 2. generally indicates an increase in DS and WL as the percentage of PCG in the blend composition increases. The DS has a peak value of $43.22 \%$ corresponding to the sample with composition (40\%PS/60\% PCG). Beyond this composition, there is an increase in WL values corresponding to the samples with compositions $(30 \% \mathrm{PS} / 70 \% \mathrm{PCG})$ to the $20 \% \mathrm{PS} / 80 \% \mathrm{PCG}$. For these two samples, swelling was initially observed before weight loss as a result of dissolution of the samples in aqueous media. For sample $20 \% \mathrm{PS} / 80 \% \mathrm{PCG}$, this dissolution is so high to give a $100 \%$ WL value. The sample with composition $100 \% \mathrm{PS} / 0 \% \mathrm{PCG}$ has a $0 \% \mathrm{DS} / \mathrm{WL}$ value indicating the absence of water sorption for the unblended PS.

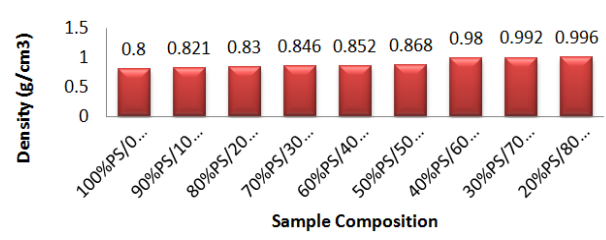

Fig 2. DS (or WL) values for various compositions of PS/PCG blends

\subsection{Mechanical Tests}

\subsubsection{Tensile Test}

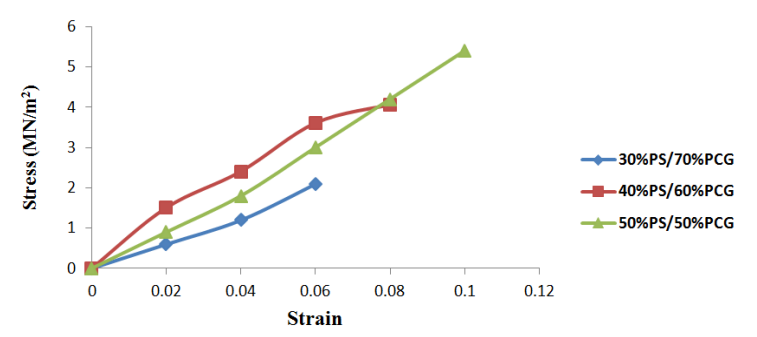

Fig 3. Stress-strain curve of $30 \% P S / 70 \% P C G, 40 \% P S / 60 \% P C G$ and $50 \% P S / 50 \% P C G$ 


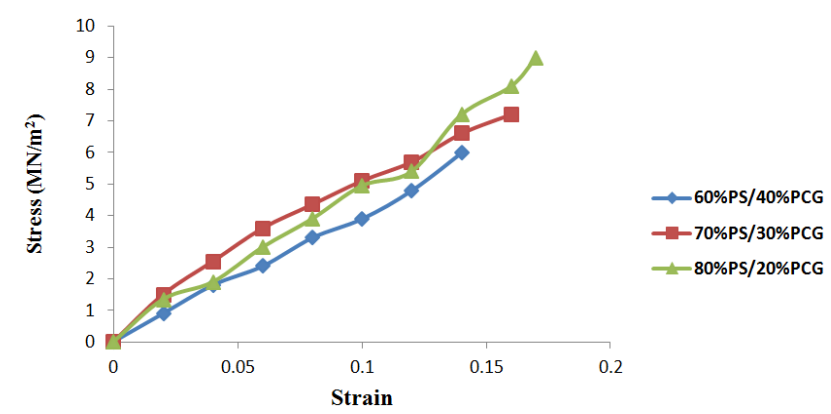

Fig 4. Stress-strain curve of $60 \% P S / 40 \% P C G, 70 \% P S / 30 \% P C G$ and $80 \% P S / 20 \% P C G$.

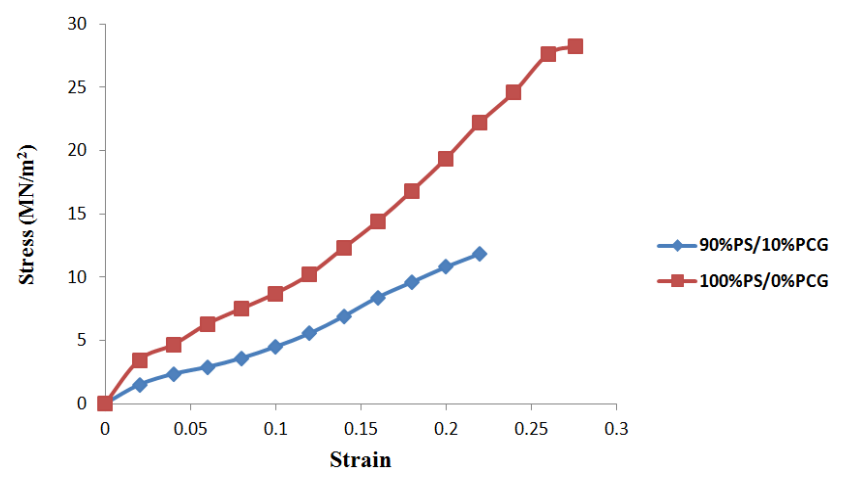

Fig 5. Stress-strain curve of $90 \% P S / 10 \% P C G$ and $100 \% P S / 0 \% P C G$.

The results of the tensile test analysis of the blended samples are summarized in figure 6 below:

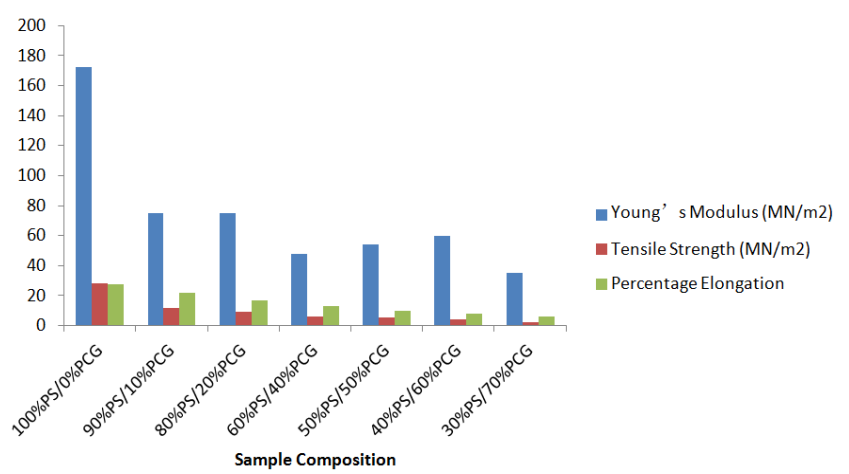

Fig 6. Plot of Young's Modulus, Tensile Strength and Percentage Elongation of PS/PCG Blend Samples.

The results of tensile tests conducted on PS/PCG blends and the control sample are shown in figures 3, 4 and 5 below. All the samples used for analysis are of the same dimension with:

Area $\left(\mathrm{A}_{\mathrm{o}}\right): 10 \mathrm{~mm} \times 4 \mathrm{~mm}=40 \mathrm{~mm}^{2}=4.00 \times 10^{-5} \mathrm{~m}^{2}$

Gauge Length $\left(\mathrm{L}_{\mathrm{o}}\right): 30 \mathrm{~mm}$

The results of tensile tests conducted on the samples are shown in figures 3 to 6 . These results indicate a general decrease in Young's modulus, tensile strength and percentage elongation with increase in the percentage of PCG in the blended samples. The decrease in Young's modulus implies a decrease in stiffness (rigidity) with increase in PCG concentration. Also the decrease in tensile strength with increase in PCG concentration is attributed to the weak intermolecular forces between the PS and PCG molecules.
The effect becomes more pronounced with increase in PCG concentration. A look at the variation of percentage elongation indicate a decrease at higher concentrations of PCG. This can be attributed to the lower elasticity of pure PCG compared to pure PS. These results indicate that the blending of PS with PCG softens PS by lowering its tensile strength. It also indicates an increase in the brittle nature of PS as a result of very low percentage elongation values at high PCG concentrations.

\subsubsection{Hardness Test Analysis of Blended Samples}

The results of hardness tests conducted on PS/PCG sample blends are shown in figure 7. Static indentation tests were conducted on the samples by the Hardness Rockwell F scale (HRF). The results indicate that the hardness of PS/PCG blends gradually increase with increase in the concentration of PCG. The control sample (100\%PS/0\%PCG) has the lowest value of $10.5 \mathrm{HRF}$ while the sample with composition $30 \% \mathrm{PS} / 70 \% \mathrm{PCG}$ has the highest hardness value of 69.1 HRF. This remarkable improvement in the hardness of PS blended samples can be attributed to the very hard nature of pure cashew tree gum in the solid state.

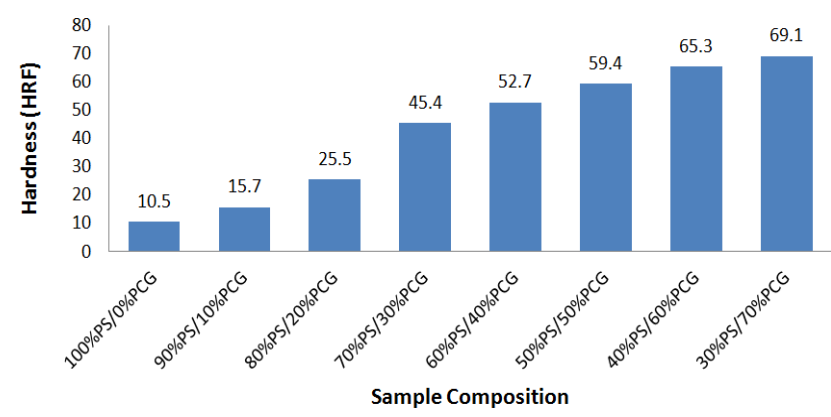

Fig 7. Plot of hardness (HRF) against sample composition of PS/PCG blend samples

\subsubsection{Charpy Impact Test Analysis of Blended Samples}

The results of charpy impact test conducted on the samples are given in figure 8 . The figure shows that sample composition $100 \% \mathrm{PS} / 0 \%$ PCG gave the highest impact strength of $5681.81 \mathrm{Jm}^{-2}$ while the sample $20 \% \mathrm{PS} / 80 \% \mathrm{PCG}$ gave the lowest value of $2272.72 \mathrm{Jm}^{-2}$. This result shows that the blending of PS with PCG decreases the impact strength of PS. Thus increase in PCG concentration lowers the toughness of PS. This general decrease with

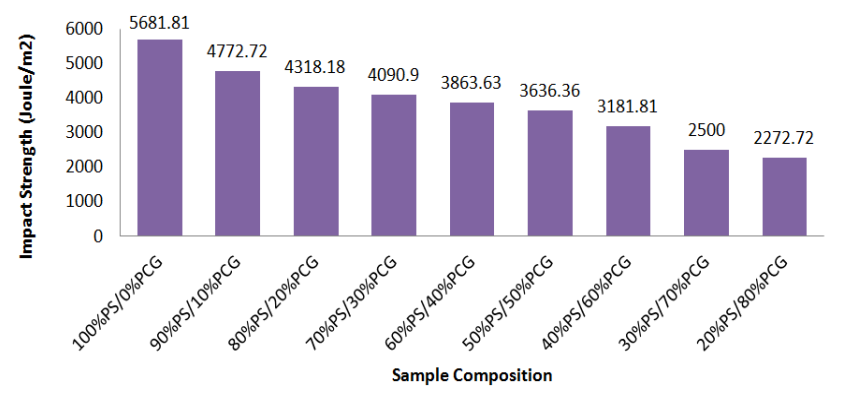

Fig 8. Plot of impact strength against sample composition for $P S / P C G$ sample blends. 


\section{Conclusion}

This research has introduced a new study of blends of polystyrene (PS) and Anacardium occidentale exudate from which the following conclusions can be drawn:

The sorption properties of PS/PCG blended samples indicated a general increase in DS and WL values with increase in the percentage of PCG in the blended samples. This trend is so pronounced that the blended PS sample disintegrated at the highest composition of PCG $(20 \% \mathrm{PS} / 80 \% \mathrm{PCG})$. The results are also consistent with the passive nature of PS to water at room temperature as indicated by the $0 \% \mathrm{DS} / \mathrm{WL}$ value of unblended PS. The water sorption behaviour exhibited by the blended samples indicate the susceptibility of these samples to hydrolytic degradation.

The density values of the blended polymer samples were observed to increase with increase in the composition of PCG in the blended samples to give a maximum value of $0.996 \mathrm{~g} \mathrm{~cm}^{-3}$. The higher density values were attributed to the higher density value of PCG compared to PS.

The tensile properties of PS such as Young's modulus, tensile strength and percentage elongation have been observed to decrease with increase in the composition of PCG. These have been attributed to weak intermolecular bonding forces between the PCG and polystyrene molecules and to the fact that PCG is glassy at ambient conditions especially in the absence of moisture.

Hardness test analysis of the samples indicate an increase in hardness with increase in PCG composition. Hence the mechanical strength of the samples increases as the percentage of PCG in the sample blends increases. The general increase in mechanical strength is due to the good hardness properties of pure PCG.

The results of the charpy impact tests conducted on the samples revealed a general decrease in impact strength with increase in PCG composition on the blended samples. A minimum value of $2272.72 \mathrm{Jm}^{-2}$ was recorded for the sample with the composition $20 \% \mathrm{PS} / 80 \% \mathrm{PCG}$ while the maximum value of $5681.81 \mathrm{Jm}^{-2}$. Thus the toughness of PS blended samples decreases with increase in PCG composition.

This research has proved the possibility of blending Polystyrene with purified cashew gum. Although, polystyrene and other related plastics are characterized by long degradation periods when discarded into the environment after use, PCG is capable of inducing biodegradation when incorporated into a plastic as given by the result of this research. The blended samples under appropriate processing conditions are capable of solving the environmental pollution problems associated with the use of polystyrene and other related plastics.

\section{References}

[1] H. Azman, W. M. Uzir, Y. C. Ching and C. H. Azahari, "Effect of Linear Low Density Polyethylene on the Mechanical Properties and Morphology of Polypropyolene/ Natural Rubber Blends, "Journal of The Institute of Materials Malaysia, vol. 4, pp. 3-18, 2003.

[2] A. I. Buthaina and M.K. Karrer, "Influence of Polymer Blending on Mechanical and Thermal Properties, "Journal of Modern Applied Science, vol. 4(9), pp. 157-161, 2010.

[3] S. Cimmino, E. Di Pace, E. Martuscelli and C. Selvestre, "Syndiotactic Polystyrene, Crystallization and Melting Behavior," Polymer, vol. 32, pp. 1080, 1991.

[4] W. Ezeagu, "Assessment of the situation and development prospects for cashew nut sector," A report, Nigerian Export Promotion Council, Abuja, Nigeria. Available online at www.unctad.org, 2002.

[5] K. E. George, J. Rani, and D. J. Francis, "Studies on NBR/PVC Blends," Journal of Applied Polymer Science, vol. 32, pp. 2867-2873, 1986.

[6] E. Gyedu- Akoto, I. Oduro, F. M. Amoah, J. H. Oldham, W. O. Ellis, K. F. Opoku-Ameyaw, and B. H. Rasheed, "PhysicoChemical Properties of Cashew Tree Gum," African Journal of Food Science, vol. 2, pp. 060-064, 2008.

[7] K. Kaniappan, and S. Latha, "Certain Investigations on the Formulation and Characterization of Polystyrene / Poly(methyl methacrylate) Blends," International Journal of ChemTech Research,vol. 3(2), pp. 708-717, 2011.

[8] R. Kumar, M. B. Patil, S. R. Patil, and M. S. Paschapur, "Evaluation of Anacardium occidentale gum as gelling agent in aceclofenac gel," International Journal of PharmTech Research, vol. 1 (3), pp. 695 - 704, 2009.

[9] A. D. McNaught, and A. Wilkinson, "IUPAC Compendium of Chemical Terminology (the "Gold Book"), 2nd ed., Blackwell Science, Oxford, 1997,"

[10] G. Scott and D. Gillead, "Degradable Polymers," Chapman \& Hall, London, 1995.

[11] F. C. Stelling, T. Huff, C. S. Speed and G. Wissler, "Structure and Properties of Rubber-Modified Polypropylene Impact Blends," Poly. Eng. Sci., vol. 26, pp. 2693, 1981.

[12] V. Wantinee, L. L. Richard and J. Jayant, "Impact Resistance of Selected Immiscible Polymer Blends," Society of Plastics Engineers [SPE] Annual Technical Conference Proceedings Cincinnati, OH May, 2007.

[13] S. M. Zebarjad, A. Lazzeri, R. Begheri, S.M. S. Rehemi and M. Frounchi, "Fracture Mechanism Under Dynamic Loading of Elastomer-Modified Polypropylene," Materials Letter, vol. 57, pp. 2733-2741, 2003. 